\title{
Estimating transmission dynamics and serial interval of the first wave of COVID-19 infections under different control measures: a statistical analysis in Tunisia from February 29 to May 5, 2020
}

Khouloud Talmoudi ${ }^{1,2 *}$, Mouna Safer ${ }^{1,2}$, Hejer Letaief ${ }^{1,2}$, Aicha Hchaichi ${ }^{1,2}$, Chahida Harizi ${ }^{3}$, Sonia Dhaouadi ${ }^{1}$ Sondes Derouiche ${ }^{1}$, Ilhem Bouaziz ${ }^{1}$, Donia Gharbi ${ }^{1}$, Nourhene Najar ${ }^{4}$, Molka Osman ${ }^{1}$, Ines Cherif ${ }^{4}$, Rym Mlallekh ${ }^{4}$, Oumaima Ben-Ayed ${ }^{4}$, Yosr Ayedi', Leila Bouabid', Souha Bougatef ${ }^{1}$, Nissaf Bouafif ép Ben-Alaya ${ }^{1,2,4}$ and Mohamed Kouni Chahed ${ }^{4}$

\begin{abstract}
Background: Describing transmission dynamics of the outbreak and impact of intervention measures are critical to planning responses to future outbreaks and providing timely information to guide policy makers decision. We estimate serial interval (SI) and temporal reproduction number ( $\left.R_{t}\right)$ of SARS-CoV-2 in Tunisia.

Methods: We collected data of investigations and contact tracing between March 1, 2020 and May 5, 2020 as well as illness onset data during the period February 29-May 5, 2020 from National Observatory of New and Emerging Diseases of Tunisia. Maximum likelihood (ML) approach is used to estimate dynamics of $\mathrm{R}_{\mathrm{t}}$.

Results: Four hundred ninety-one of infector-infectee pairs were involved, with $14.46 \%$ reported pre-symptomatic transmission. SI follows Gamma distribution with mean 5.30 days [95\% Confidence Interval (Cl) 4.66-5.95] and standard deviation 0.26 [95\% Cl 0.23-0.30]. Also, we estimated large changes in $R_{t}$ in response to the combined lockdown interventions. The $R_{t}$ moves from 3.18 [95\% Credible Interval (Crl) 2.73-3.69] to 1.77 [95\% Crl 1.49-2.08] with curfew prevention measure, and under the epidemic threshold ( 0.89 [95\% Crl 0.84-0.94]) by national lockdown measure.
\end{abstract}

Conclusions: Overall, our findings highlight contribution of interventions to interrupt transmission of SARS-CoV-2 in Tunisia.

Keywords: Coronavirus, Reproduction number, Serial interval, Lockdown, Statistical models, Tunisia

\footnotetext{
* Correspondence: talmoudi.khouloud@gmail.com

'National Observatory of New and Emerging Diseases, Tunis, Tunisia

${ }^{2}$ Research laboratory "Epidemiology and Prevention of Cardiovascular Diseases in Tunisia", Tunis, Tunisia

Full list of author information is available at the end of the article
}

C C The Author(s). 2020 Open Access This article is licensed under a Creative Commons Attribution 4.0 International License, which permits use, sharing, adaptation, distribution and reproduction in any medium or format, as long as you give appropriate credit to the original author(s) and the source, provide a link to the Creative Commons licence, and indicate if changes were made. The images or other third party material in this article are included in the article's Creative Commons licence, unless indicated otherwise in a credit line to the material. If material is not included in the article's Creative Commons licence and your intended use is not permitted by statutory regulation or exceeds the permitted use, you will need to obtain permission directly from the copyright holder. To view a copy of this licence, visit http://creativecommons.org/licenses/by/4.0/ The Creative Commons Public Domain Dedication waiver (http://creativecommons.org/publicdomain/zero/1.0/) applies to the data made available in this article, unless otherwise stated in a credit line to the data. 


\section{Introduction}

Since December 2019, the epidemic of the novel severe acute respiratory syndrome coronavirus 2 (SARS-CoV2 ), the causative agent of coronavirus disease 2019 (COVID-19) has been spreading. Initially present in Wuhan, China [1], it was officially declared a pandemic on March 11, 2020 by the World Health Organization [2].

In Tunisia, as of January 22, 2020, government has implemented early prevention measure, including screening in point of entry and systematic 14 days isolation of travelers returning from risk areas. The first confirmed case, among an international traveler from Italy, was reported on March 2, 2020. One week later, Tunisian government reinforce its suppression strategy with additional preventive measures. Following the reporting of 13 new cases on March 12, 2020 closure of school and university facilities was announced. The government announced further prevention measures, specifically border closure with Italy as of 14 March. On March 17, 2020, a curfew throughout the whole country, starting on March 18, 2020 was decided. Also, the closure of all sea and air borders were applied as of March 18, 2020. On March 20, a national lockdown, with a ban of transport between governorates were announced from 22 March. Finally, transition to the risk level 3 was announced on March 22, 2020.

Data are accumulating daily on transmission of COVID-19 virus in Tunisia. These data are vitally important in controlling the spread of this virus and settle the current pandemic. Determination of the serial interval (SI), the time between the symptoms onset in the primary patient (infector or index case) and symptoms onset in the patient receiving that infection from the infector (the infectee or secondary case) is fundamental in estimating the basic reproduction number $\left(R_{0}\right)$, which is the number of infectees resulting from one infector throughout his entire infectious period [3, 4].

Besides, the temporal reproduction number $R_{t}$ is one of the key parameters in public health because it determines the extent of the epidemic, i.e. it characterizes the number of infected people caused by a contagious person during the period of his infection; it summarizes the potential transmissibility of the disease and indicates whether an epidemic is under control.

Up to now, the reproduction number $\left(R_{t}\right)$ can only been estimated retrospectively for periods from which all secondary cases had been detected. In terms of policy making and evaluation during outbreaks, obtaining estimates of the temporal tendency in the reproduction number covering as recent a time as possible would be critical [5].

$R_{t}$ is the only reproduction number easily estimated in real time [6]. Moreover, effective control measures undertaken at time $t$ are expected to result in a sudden decrease in $\mathrm{R}_{\mathrm{t}}$. Hence, assessing the impact of public health interventions to mitigate the disease is easier by using estimates of $\mathrm{R}_{\mathrm{t}}$. For these reasons, we focus on estimating the instantaneous reproduction number $R_{t}$ in Tunisia.

\section{Material and methods}

Data

Data of SARS-CoV-2 were collected from the National Observatory of New and Emerging Diseases of Tunisia. The first dataset consists of time series of symptom onset reported from February 29, 2020, to May 5, 2020. The second dataset is obtained from contact tracing between March 1 and May 5, 2020. It was screened to clearly identified transmission events, which are a known pairs of index and secondary cases and the dates of symptom onset for both cases. Data were anonymized for this study, we only report the certain pairs of infector/infectee during the study period.

\section{Inference methods}

A two-step procedure is used to estimate the Rt. It consists of the use of data informing the SI and daily temporal incidence onset of cases data [7]. The first step uses data on known pairs of index (infector) and secondary (infectee) cases to estimate the SI distribution; the second step estimates the time-varying reproduction number jointly from disease onset time series and from the SI distribution fitted in the first step.

\section{Estimation of the serial interval distribution}

Serial intervals distribution can be estimated during an ongoing outbreak using data from the list of censored lines by interval, i.e. the lower and upper limits of the date of symptom onset in index and secondary cases [8]. For each infector/infectee pair, a delay between the date of symptom onset, as claimed by the infector, and the date of symptom onset, as claimed by the infected person, is calculated [9]. In some cases, the infected person develops symptoms before the person transmitting the virus, in this case the difference between two dates will be negative.

Maximum likelihood (ML) estimates and the Akaike information criterion (AIC) are used to evaluate widely used parametric candidate models for the SARS-CoV-2 serial interval distributions namely normal, lognormal, Weibull, and gamma. Since our SI data includes a considerable number of non-positive values, we fit the four distributions both to positive values (truncated) and to shifted data, in which 12 delays are added to each observation [9]. However, caution against making assessments and projections 
based on the truncated data should be carefully explored and we do not believe there is cause for excluding the non-positive data.

\section{Estimation of the number of temporal reproduction}

At the beginning of an epidemic, when the whole population is susceptible (i.e. not immune), this number takes on a particular value denoted $R_{0}$ and called basic reproduction number [10].

The calculation of $R_{0}$ is based on three underlying assumptions as follows:

1. Screening strategy in Tunisia is assumed to be constant,

2. Spatial structure is neglected,

3. Incidences used are those available since February 29, 2020 and until March 18, 2020 (date of the curfew) for $\mathrm{R}_{0}$ and until 5 May 2020 for temporal reproduction number.

During the outbreak, when the proportion of immunized persons becomes sufficiently large to slow the transmission of the virus (by an effect similar to a reduction in the number of individuals still susceptible), we speak about the effective, or temporal, reproduction number denoted $\mathrm{R}_{\mathrm{t} \text {. }}[11]$

Analyses for estimating the reproduction number were conducted using the EpiEstim [7, 12, 13] package on the $R$ statistical software (version 3.6.3) [14]. This package is based on an approach that is motivated by the fact that in the situation where the epidemic under study would still be ongoing, and more particularly when it comes to evaluating the effectiveness of control measures, the total number of infections caused by the latest cases detected is not yet known. For EpiEstim package, the highlighted approach to temporal reproduction number leads to the instantaneous reproduction number, which is prospective: its calculation is based on the potential number of secondarily infected persons that a cohort of cases could have caused if the conditions of transmissibility had remained the same as at the time of their detection.

Let's denote the total cases by symptom onset arising at time-step $t$ by $\mathrm{I}_{\mathrm{t}}$ (assuming total cases of local and imported). Following $[6,7]$, in which the timedependent reproduction number, $R_{t}$, is illustrated as the ratio of the number of new infected cases at time $t, I_{t}$, and the total infection potential across all infected individuals at time unit $\mathrm{t}, \Lambda_{\mathrm{t}}$. If there is a single serial interval distribution $\omega_{\mathrm{s}} \quad(\mathrm{s}=1,2, \ldots)$, representing the probability of a secondary case arising a time period $\mathrm{s}$ after the primary case, each incident case that appeared at a previous time-step $t-s$ contributes to the current infectiousness at a relative level given by $\omega_{s}$. Therefore conditional on $\omega_{s}, \Lambda_{t}$ can be computed as follows:

$$
\Lambda_{\mathrm{t}}\left(\mathrm{w}_{\mathrm{s}}\right)=\sum_{\mathrm{s}=1}^{\mathrm{t}} \mathrm{I}_{\mathrm{t}-\mathrm{s}} \mathrm{w}_{\mathrm{s}}
$$

Formally the EpiEstim package maximizes the likelihood of incidence data (seen as a Poisson count) observed over a time window of size $\tau$ ending at $t$. The assumption made here is that the reproduction number is constant over this time window $[t-\tau, t]$. The estimation of the reproduction number at each time window, denoted $R_{t, \tau}$, for the time interval $[t-\tau, t]$ verifies:

$$
R_{t, \tau}(t)=\underset{R_{t}}{\operatorname{argmax}} \prod_{k=t-\tau}^{t} \frac{\left(R_{t} \Lambda_{k}\left(w_{s}\right)\right)^{I_{k}} \exp \left(-R_{t} \Lambda_{k}\left(w_{s}\right)\right)}{I_{k} !}
$$

Thereby, an estimation of $R_{t}$ is obtained given both the incidence and serial interval data, from which the mean and $95 \%$ intervals of $R_{t}$ can be computed. The proposed formula may also be used for early detection of the effect of control measures to prevent the spread of the virus on the incidence of new cases.

For the next parts of the document, $R_{t}$ is denoted $R$ for simplicity. If $R>1$, then one person infects more than one person, on average and the epidemic is growing. As the epidemic spreads, $R$ decreases as an increasing proportion of the population becomes immune. When the threshold for group immunity is exceeded, $\mathrm{R}$ drops below 1, an epidemic peak is reached and the epidemic decreases. Public health control measures can also decrease $\mathrm{R}$ and thus reach epidemic peak before the threshold of population immunity is reached. Therefore, knowing the value of $\mathrm{R}$ at time $\mathrm{t}$ is essential to determine the status of the epidemic.

Moreover, the overall infectivity due to previously infected individuals of an outbreak at time $t$, denoted $\lambda_{t}$, is a relative measure of the current force of infection. It is calculated as the sum the previously infected individuals $I_{t}$, weighted by their infectivity at time $t$ and is given by:

$$
\lambda_{t}=\sum_{k=1}^{t-1} I_{t-k} w_{k}
$$

The critical parameter for these calculations is the distribution of SI. If $\lambda$ is falling, then that's good: if not, bad.

\section{Results}

\section{Distribution of serial interval}

Contact tracing data, collected in the study period (between Feb 29 and May 5, 2020) included 188 unique infectors, with 117 index cases (infectors) who infected 
multiple people and 39 individuals that appear as both infector and infectee. Notably, 71 of the 491 (14.46\%) reported cases have negative values in number of days separating symptom onset date for the infector and symptom onset date for the infected person. This indicates that the infected case developed symptoms earlier than the infector. Thus, the results suggest contamination during the asymptomatic phase or pre-symptomatic phase of the infected source may be occurring, i.e., infected persons may be infectious before their symptoms appear.

The SI estimates by fitting the four parametric distributions both to positive values (truncated) and to shifted data show that the gamma distribution provides the best fit for the truncated data (followed closely by the Weibull and lognormal). Fitted distributions can be found in the Additional file: Fig. S1 and Table S1. Also, the SI was estimated using the full dataset. Again, the gamma distribution can provide the best fit for the full dataset (shifted or truncated) and thus is the distribution we recommend for future epidemiological assessments and planning. Results from gamma distribution estimated mean SI of 5.30 days
[95\% CI 4.66-5.95] with a standard deviation (SD) of 0.26 [95\% CI 0.23-0.30] for SARS-CoV-2 in Tunisia.

\section{Calculating $\mathbf{R}$}

As of May 5, 2020, 1028 SARS-CoV-2 symptom onset data were reported with 246 (23.9\%) being imported. In Tunisia, the epidemic started with one imported case from Italy, followed by cases with travel history and known contacts with imported cases (Fig. 1a). In March (week 1 to mid-5), the largest part of cases were local with no traveling activity (353 of 562 , or $63 \%$ ). For this wave of the epidemic (Fig. 1b), the R for total cases was above the epidemic threshold $(>1)$. For each day $t$ of the epidemic, we estimated weekly window $\mathrm{R}$ ending on that day (Fig. 1b). Estimates are not shown from the very beginning of the epidemic because precise estimation is not possible in this period. $\mathrm{R}$ initially increased from a median value of 2.06 [95\% CrI 1.36-2.97] early in the second week to 3.46 [95\% CrI 2.70-4.35] at the end of the same week. $\mathrm{R}$ increased in the third week, reaching a peak of 5.01 [95\% CrI 4.03-6.13] in the beginning of this

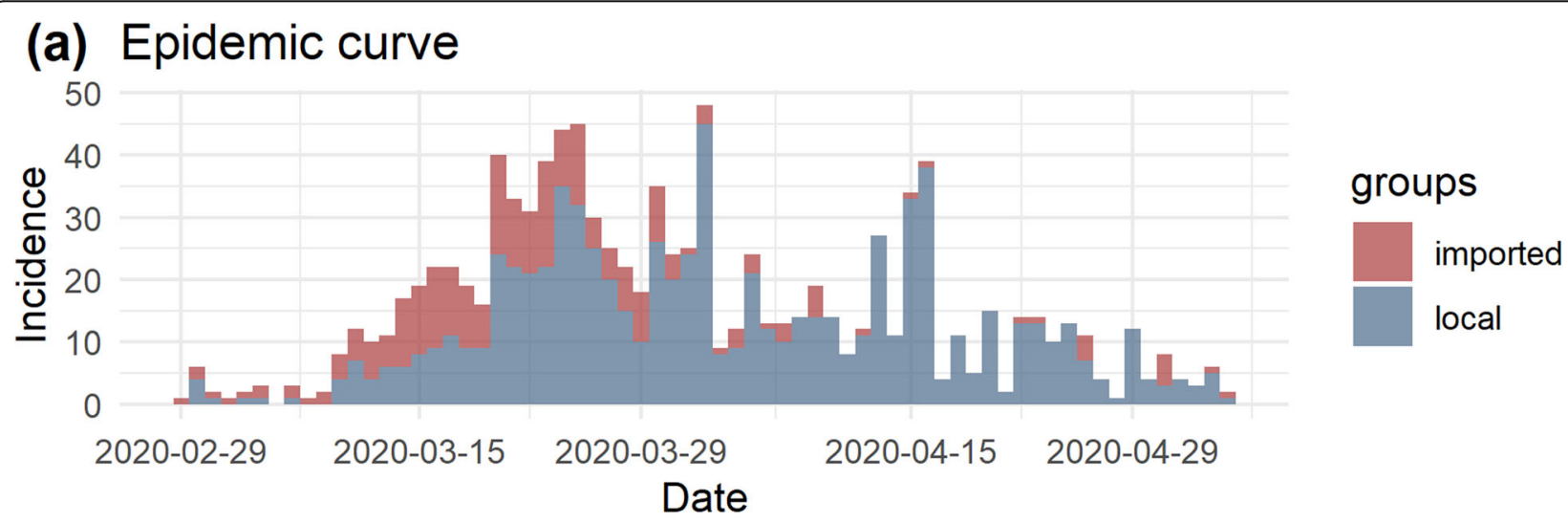

\section{(b)Estimated R}

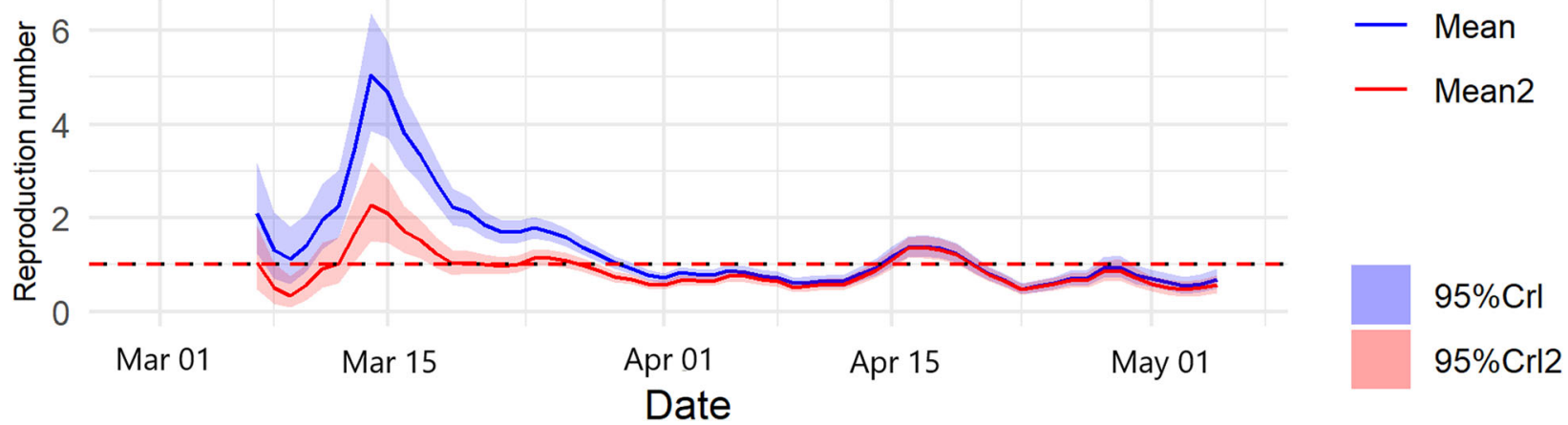

Fig. 1 Instantaneous effect reproduction number for SARS-CoV-2 by symptom onset date in Tunisia. In the first graph is shown the daily symptom onset time series for coronavirus from February 29, 2020-May 5, 2020. The second graph shows the estimated reproduction number over sliding weekly windows (posterior mean and 95\% credible interval, with estimates for a time window plotted at the end of the time window); the blue color is for all cases and the red color is for local cases; the solid lines show the posterior means and the transparent zones show the $95 \%$ credible intervals; the horizontal dashed red line indicate the threshold value $\mathrm{R}=1$ 
week. Note that in this wave the estimated reproduction number for local cases (estimated as 2.25 [95\% CrI 1.623.03] in the beginning of week 3 ) is, as expected, much lower than estimated when assuming that all cases were linked by local transmission (estimated as 5.01 [95\% CrI 4.03-6.13]). The increased values of $R$ from weeks 2 to 3 for both all cases and local cases suggests increasing transmissibility. This may be suggested by the existence of early "superspreaders".

At the start of week 4, R decreased to 1.69 [95\% CrI 1.49-1.90]. In April (mid-week 5 to 9), almost all cases had local transmission with few travel history (413 of 450 , or $92 \%)$. $\mathrm{R}$ continue to decrease till the middle of week 7 with values under the epidemic threshold $(<1)$. Then, it increased again (mid-week 7 to mid 8) up to 1.40 [95\% CrI 1.21-1.60] by 17 April 2020. Finally, R decreased again to the end of period study, up to 0.68 [95\% CrI 0.52-0.87]. The weekly estimates of $\mathrm{R}$ can be found in the Additional file: Table S2. This could reflect the impact of control measures or could be due to the depletion of susceptibles in the Tunisian population.

Besides, to reinforce our findings and provide more information on the epidemic situation in Tunisia, the overall infectivity is globally increased over the first period study, from February 29, 2020 to April 8, 2020, then it remains stable by April 18, 2020 to peak again between April 22 to April 24, 2020. Finally, it decreases during the last period study. Illustration of the overall infectivity can be found in the Additional file: Fig. S2.

Impact of curfew and lockdown prevention measures on $\mathbf{R}$ In Tunisia, the curfew was applied on March 18, 2020 and the lockdown was applied on March 22, 2020. We estimated large changes in $\mathrm{R}$ in response to the combined lockdown interventions. Our results suggest that the lockdown was effective in terms of reducing transmissibility (Fig. 2), as the estimated reproduction number during the lockdown was significantly lower compared to pre-intervention period. The $\mathrm{R}$ moves from 3.18 [95\% CrI 2.73-3.69] to 1.77 [95\% CrI 1.49-2.08] with curfew prevention measure, meaning that it reduces transmissibility but the risk of contagion is still alarming. By national lockdown measure, this value moves to 0.89 [95\% CrI 0.84-0.94] (<1), indicating the substantial impact of this prevention measure in reducing transmission of the epidemic.

\section{Discussion}

We analyzed the transmission dynamics of SARS-CoV-2 infection in Tunisia in the first 3 months of the epidemics where all prevention measures were implemented, especially curfew and national lockdown.

Our results focus on the use of the likelihood-based method to estimate initial $R_{t}$ and SI. Utilizing temporal symptom onset data and contact tracing, we provide estimates of the transmissibility parameters of SARS-CoV-2 during the first wave experienced in Tunisia. These data are incorporated to provide robust estimates of transmission parameters.

Our estimates of the SI for SARS-CoV-2 in Tunisia better resemble a gamma distribution with an estimated mean of 5.30 days [95\% CI 4.66-5.95]. These estimates of the mean SI are higher than the published estimates of 4.6 days [ $95 \% \mathrm{CI}: 3.5,5.9$ ] among 18 certain pairs calculated at early stage of the COVID-19 epidemic [4], indicating that SARS-CoV-2 infection in Tunisia leads to slow cycles of transmission from one generation of cases to the next. Recent estimates for the mean serial interval of COVID-19 range from 3.9 days [95\% CI 2.7-73] [15] and 4.0 days [95\% CI 3.1-4.9] [4] to 7.5 days [95\% CI

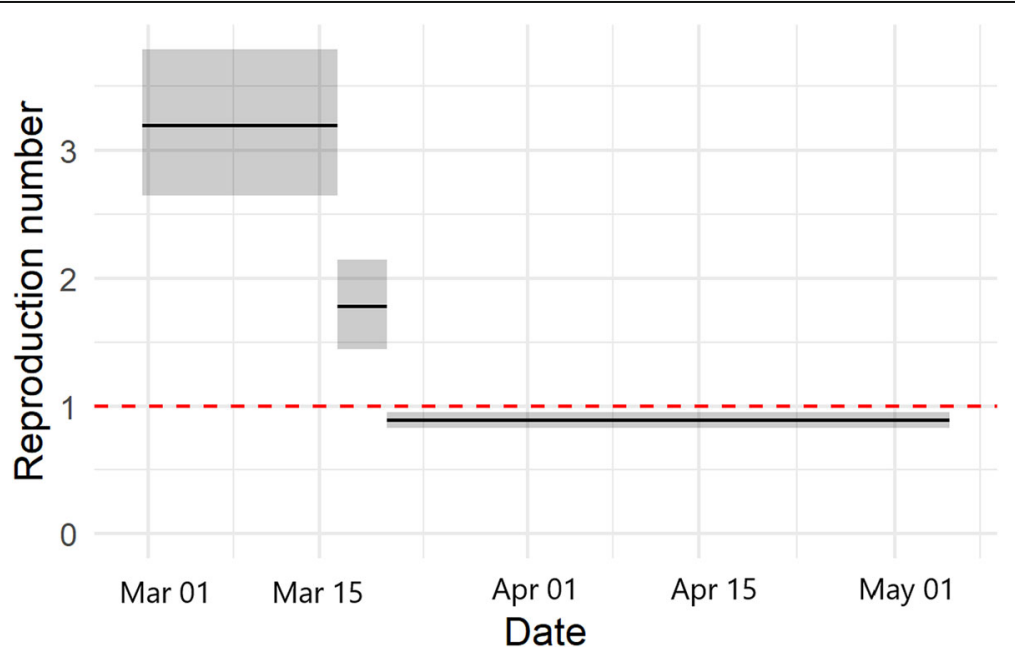

Fig. 2 Impact of interventions on the estimates of the reproduction number R during the study period in Tunisia. The first intervention was the curfew which is applied on March 18, 2020 and the second one was the national lockdown applied on March 22, 2020 
5.3-19] [3] based on data from 21, 28 and 6 pairs, respectively. Also, the mean SI in Tunisia is considerably lower than reported mean serial intervals of 8.4 days for SARS [16] and 12.6 days [17] - 14.6 days [18] for MERS. This indicates that calculations made using the SARS SI may introduce bias.

At least, two sources of bias can be potentially considered in our estimates, which are likely to cause underestimation of SARS-CoV-2 serial intervals. First, the distribution of SI varies during an epidemic, with the time separating successive cases close to the epidemic peak [9]. To provide insight, a susceptible person would probably become infected more quickly if he is surrounded by more than one infected person. Since our estimates are based on transmission cases reported during the early stages of outbreaks, such compression are not explicitly accounted and we interpret the estimates as basic serial intervals at the beginning of an epidemic. However, our estimates may reflect effective serial intervals when certain of the reported infections occurred in the amidst of growing clusters, that would be expected during a period of epidemic growth. Second, the dates of symptom onset of each infector was likely based on individual remembrance of past activities. If accuracy of the recall is impeded by time, it is highly possible that recent encounters (short serial intervals) are attributable to infected cases rather than over past encounters (longer serial intervals). This information is self-reported from infected cases. Therefore, an information or reporting bias may occur. In contrast, the reported serial intervals may be biased upwards by travel-related delays in transmission from primary cases that were infected in another countries before returning in Tunisia. In that case, if their infectious period began while still traveling, then it is very unlikely to observe early transmission events with shorter serial intervals. Given the diversity in type and reliability sources of bias when estimating the SI, particular cautions to our findings should be granted. Our results provide working hypotheses regarding the infectivity of coronavirus in Tunisia, which will need to be validated as new data become available. In our study, we used the symptom onset dates for both infector and infected confirmed cases. However, the last exposure dates to infected person(s) for pairs of infector/infectee were not considered in this study due to messing data. These dates are useful to settle if infection is occurring during the asymptomatic phase of the infection. However, negative values in delays separating symptom onset date for the infector and symptom onset date for the infected person strongly suggest infection occurring during the asymptomatic phase of SARS-CoV-2 in Tunisia. Although contamination from asymptomatic persons has not been proven before, recent studies have highlighted the existence of such transmission [19, 20]. However, contamination from asymptomatic persons lower in comparison with symptomatic cases [21]. Here, the potential impacts for the control of SARS $\mathrm{CoV}-2$ are mixed. While our lower estimates for $\mathrm{R}$ suggest easier lockdown, the asymptomatic transmission events remains a concern. Future works are developed to provide more information of the contamination of SARS-CoV-2 during the asymptomatic phase of the infected sources in Tunisia.

In Tunisia massive prevention strategies have been applied at early stage of the SARS-CoV-2 epidemic. These strategies include implementing travel restrictions, isolation of infected individuals, active contact tracing, make quarantining compulsory, and enforcing lockdown [22]. In Tunisia, where all interventions were implemented in short time period and some of them were separated by a short time interval, these individual effects are by definition unidentifiable. Despite this, while individual impacts cannot be determined, their estimated joint impact is strongly empirically justified [23]. Our results suggest that the lockdown was effective in mitigating transmissibility of the disease, as the estimated reproduction number during the lockdown was significantly lower compared to initial-intervention period. We showed that after less than 5 weeks from the beginning of the epidemic, $R$ dropped below the epidemic threshold. This corresponds to a decline of new cases, which is the result of herd immunity altogether with public health control measures. However, despite the dependence of infectious diseases on climate factors [24], there is no evidence so far supporting the impact of warmer climate factors in reducing the transmissibility of the disease [25].

Likewise, we estimated that there was an early decrease in $R$ for the outbreak of coronavirus in Tunisia. But just estimating $\mathrm{R}$ does not allow us to determine whether this reflects a true reduction in transmissibility, possibly due to the national lockdown or the depletion of susceptibles. Furthermore, with $\mathrm{R}$ values dropping significantly, the acquisition rate of herd immunity will slow down rapidly. This indicates the ability of the virus to spread rapidly should interventions be lifted. Interestingly, just after the first peak of incidence, $R$ decreased under 1 for almost 2 weeks, raised above 1 for 5 days, and decreased again under 1 , indicating that the epidemic was not yet over; and indeed, a second peak was still to come.

Some limitations in this study can be highlighted. First, we presume all infected cases are known and consistently reported over the study. Violation of this assumption may lead to biased estimates of the SI and the reproduction number [26]. Similarly, the variation in case tracking over time may bias our estimates of the time variation of $R_{t}$. In general, higher reporting rates can be expected in the early phase of the epidemic, with 
reporting fatigue becoming a factor in late phase. Second, our estimates do not incorporate regional heterogeneity probably existing in transmission patterns, or to assess its impact on overall measures of reproduction number.

Despite these limitations, the post-pandemic estimates reported in this study add to general acquisition and knowledge of coronavirus transmissibility parameters, which were previously dominated by estimates from other countries, or occasionally from other epidemics, and are often based on preliminary data. It remains important that the revision of the parameters, based on exhaustive data from different geographical scales, be integrated into the planning of mitigation strategies for future pandemics. Nevertheless, the methods used in this study would be adaptable to generate real-time estimates for future epidemics. As we continue to build epidemiological capacity in our country, urgent improvements need to be implemented such as, digitizing contact tracing, the need for rapid assessments of transmissibility of novel pathogens, in addition to disease severity, to better inform public health interventions.

\section{Supplementary Information}

Supplementary information accompanies this paper at https://doi.org/10. 1186/s12879-020-05577-4.

Additional file 1: Figure S1. Maximum likelihood distributions fit to transformed COVID-19 serial intervals (491 reported transmission events in Tunisia between March 1, 2020 and May 5, 2020). To evaluate several positive-valued distributions (lognormal, gamma and Weibull), we took two approaches to addressing the negative-valued data. First, we left truncated the data (i.e., removed all non-positive values) for (A) all infection events. Second, we shifted the data by adding 12 days to each reported serial interval for (B) all infection events. Table S1. Model comparison for COVID-19 serial intervals based on all 491 reported transmission events in Tunisia between March 1, 2020 and May 5, 2020. Table S2. Weekly window reported estimates of the reproduction number (R) during the study period in Tunisia. Figure S2. Overall infectivity between February 29, 2020 and May 5, 2020.

\section{Abbreviations}

AIC: Akaike information criterion; Cl: Confidence Interval; Crl: Credible interval; COVID-19: Causative agent of coronavirus disease 2019; ML: Maximum likelihood; $R_{0}$ : Basic reproduction number; $R_{t}$ : Temporal reproduction number; SARS-CoV-2: Severe acute respiratory syndrome coronavirus 2; SD: Standard deviation; SI: Serial interval

\section{Acknowledgments}

We acknowledge the health teams involved in investigation and case tracing, admin team of National observatory of new and emerging diseases of Tunisia, the field teams, members of the regional monitoring units, and all lab team contributing to the laboratory diagnosis based on the real-time RTPCR test. The authors thank World Health Organization (WHO) office of Tunis, Tunisia for assistance with data collection. We acknowledge Dryad for publishing the datasets [27].

\section{Authors' contributions}

$M C$ and NBA conceived the study. KT, MS, NBA, MC carried out the analysis. $\mathrm{MS}$ and $\mathrm{HL}$ collected and managed contact tracing. HA and NBA checked and validated symptom onset data. KT drafted the first manuscript. MS, AH and SD provided helpful information. CH, IB, DG, NN, MO, IC, RM, OB-A, YA,
LB, SB, [SD]1 and [SD]2 participated in data collection and insured contact tracing. NBA and MC provided guidance and carefully revised the manuscript. All authors discussed the results, critically read and revised the manuscript, and gave final approval for publication.

\section{Funding}

This research did not receive any specific grant from funding agencies in the public, commercial, or not-for-profit sectors.

\section{Availability of data and materials}

The datasets generated and/or analyzed in this study are not publicly available for respect for patient privacy, but are available from the corresponding author upon reasonable request.

\section{Ethics approval and consent to participate}

The follow-up data of individual patients were collected from National Observatory of New and Emerging Diseases of Tunis, Tunisia. Administrative permissions were required to access and use the meta-data described in our study. Permission was granted from the National Observatory of New and Emerging Diseases of Tunis, Tunisia. Data were anonymized for this study. Neither ethical approval nor individual consent was not applicable.

\section{Consent for publication}

Not applicable.

\section{Competing interests}

Authors declare no conflict of interest.

\section{Author details}

${ }^{1}$ National Observatory of New and Emerging Diseases, Tunis, Tunisia. ${ }^{2}$ Research laboratory "Epidemiology and Prevention of Cardiovascular Diseases in Tunisia", Tunis, Tunisia. 'Department of Epidemiology and Statistics, Abderrahman Mami Hospital, Ariana, Tunisia. ${ }^{4}$ Department of Epidemiology and Public Health, Faculty of Medicine of Tunis, Tunis El Manar University, Tunis, Tunisia.

Received: 23 May 2020 Accepted: 3 November 2020

Published online: 02 December 2020

\section{References}

1. Chinese Center for Disease Control and Prevention. Epidemic update and risk assessment of 2019 novel coronavirus 2020; 2020. [Available from: http://www.chinacdc.cn/yyrdgz/202001/P020200128523354919292.pdf]. Accessed 18 Feb 2020).

2. World Health Organization. Statement on the second meeting of the international health regulations (2005) emergency committee regarding the outbreak of novel coronavirus (2019-nCoV); 2020. Available from: https:// www.who.int/news-room/detail/30-01-2020-statement-on-the-secondmeeting-of-the-international-health-regulations-(2005)-emergencycommittee-regarding-the-outbreak-of-novel-coronavirus-(2019-ncov)]. Accessed 18 Feb 2020.

3. Li Q, Guan X, Wu P, Wang X, Zhou L, Tong Y. Early transmission dynamics in Wuhan, China, of novel coronavirus-infected pneumonia. N Engl J Med. 2020;382(13):1199-207.

4. Nishiura $H$, Linton NM, Akhmetzhanov AR. Serial interval of novel coronavirus (COVID-19) infections. Int J Infect Dis. 2020;93:284-6.

5. Cauchemez S, Boëlle PY, Donnelly CA, Ferguson NM, Thomas G, Leung GM, et al. Real-time estimates in early detection of SARS. Emerg Infect Dis. 2006; 12:110-3.

6. Cori A, Nouvellet P, Garske T, Bourhy H, Nakouné E, Jombart T. A graphbased evidence synthesis approach to detecting outbreak clusters: an application to dog rabies. PLoS Comp Biol. 2018;14:e1006552.

7. Thompson RN, Stockwin JE, van Gaalen RD, Polonsky JA, Kamvar ZN, Demarsh PA, et al. Improved inference of time-varying reproduction numbers during infectious disease outbreaks. Epidemics. 2019;29:100356.

8. Cowling BJ, Fang VJ, Riley S, Peiris JM, Leung GM. Estimation of the serial interval of influenza. Epidemiology. 2009;20:344-7.

9. Du Z, Xu X, Wu Y, Wang L, Cowling BJ, Meyers L. Report: the serial interval of COVID-19 from publicly reported confirmed cases. Emerg Infect Dis. 2020; 26(6):1341-3. 
10. Modeling group of the ETE team (MIVEGEC Laboratory, CNRS, IRD, University of Montpellier). Report: estimation du nombre de reproduction temporel; 2020. [Available from: http://bioinfo-shiny.ird.fr:3838/Rt/]. Accessed 17 Apr 2020.

11. Cori A, Ferguson NM, Fraser C, Cauchemez S. A new framework and software to estimate time-varying reproduction numbers during epidemics. Am J Epidemiol. 2013;178(9):1505-12.

12. Cori A, Cauchemez S, Ferguson NM, Fraser C, Dahlqwist E, Demarsh PA, et al. EpiEstim: estimate time varying reproduction numbers from epidemic curves. R package version: 2.2-1; 2019. [Available from: https://CRAN.Rproject.org/package=EpiEstim]. Accessed 8 Jul 2019.

13. Wallinga J, Teunis P. Different epidemic curves for severe acute respiratory syndrome reveal. Am J Epidemiol. 2004;160(6):509-16.

14. R Development Core Team. R: a language and environment for statistical computing. R Foundation for statistical computing, Vienna, Austria; 2017. [Available from: http://www.r-project.org].

15. Zhao S, Gao D, Zhuang Z et al. Estimating the serial interval of the novel coronavirus disease (COVID-19): A statistical analysis using the public data in Hong Kong from January 16 to February 15, 2020, 13 May 2020, PREPRINT (Version 2). [Available from: https://doi.org/10.21203/rs.3.rs-18805/v2].

16. Lipsitch M, Cohen T, Cooper B, Robins JM, Ma S, James L, et al. Transmission dynamics and control of severe acute respiratory syndrome. Science. 2003; 300(5627):1966-70.

17. Cowling BJ, Park M, Frang VJ, Wu P, Leung GM, Wu JT. Preliminary epidemiological assessment of MERS-Cov outbreak in South Korea. Eurosurveillance. 2015;20(25):7-13.

18. Park SH, Kim Y-S, Jung Y, Choi SY, Cho N-H, Jeong HW, et al. Outbreaks of Middle East respiratory syndrome in two hospitals initialed by a single patient in Daejeon, South Korea. Infect Chemother. 2016;48(2):99-107.

19. World Health Organization. Transmission of SARS-CoV-2: implications for infection prevention precautions; 2020. [Available from: https://www.who. int/news-room/commentaries/detail/transmission-of-sars-cov-2-implicationsfor-infection-prevention-precautions]. Accessed 9 July 2020.

20. Wei WE, Li Z, Chiew CJ, Yong SE, Toh MP, Lee VJ. Presymptomatic transmission of SARS-CoV-2_Singapore, January 23-march 16, 2020. Morb Mortal Wkly Rep. 2020;69(14):411.

21. Oran DP, Topol EJ. Prevalence of asymptomatic SARS-CoV-2 infection: a narrative review. Ann Intern Med. 2020;173(5):362. https://doi.org/10.7326/ M20-3012.

22. Fang Y, Nie Y, Penny M. Transmission dynamics of the COVID19 outbreak and effectiveness of government interventions: a data-driven analysis. J Med Virol. 2020;92:645-65.

23. Flaxman S, Mishra S, Gandy A, et al. Report 13: Estimating the number of infections and the impact of non-pharmaceutical interventions on COVID19 in 11 European countries. Imperial College COVID-19 Response Team 2020. doi: https://doi.org/10.25561/77731.

24. Talmoudi K, Bellali H, Ben-Alaya N, Saez M, Malouche D, Chahed MK. Modeling zoonotic cutaneous leishmaniasis incidence in Central Tunisia from 2009-2015: forecasting models using climate variables as predictors. PLoS Negl Trop Dis. 2017;11(8):e0005844.

25. Xie J, Zhu Y. Association between ambient temperature and COVID-19 infection in 122 cities from China. Sci Total Environ. 2020;724(2020):138201

26. White LF, Pagano M. Reporting errors in infectious disease outbreaks, with an application to pandemic influenza a/H1N1. Epidemiol Perspect Innov. 2010;7:12.

27. Talmoudi K., Safer M, Letaief H, Hchaichi A, Harizi C, Dhaouadi S et al. Data from: estimating transmission dynamics and serial interval of the first wave of COVID-19 infections under different control measures: a statistical analysis in Tunisia from February 29 to may 5, 2020. Dryad. 2020. doi: https://doi. org/10.5061/dryad.b8gtht799.

\section{Publisher's Note}

Springer Nature remains neutral with regard to jurisdictional claims in published maps and institutional affiliations.

Ready to submit your research? Choose BMC and benefit from:

- fast, convenient online submission

- thorough peer review by experienced researchers in your field

- rapid publication on acceptance

- support for research data, including large and complex data types

- gold Open Access which fosters wider collaboration and increased citations

- maximum visibility for your research: over $100 \mathrm{M}$ website views per year

At BMC, research is always in progress.

Learn more biomedcentral.com/submissions 